\title{
A Configurable Smart E-Nose for Spatio-Temporal Olfactory Analysis
}

\author{
Carlos Sanchez-Garrido, Javier G. Monroy and Javier Gonzalez-Jimenez \\ Dept. of System Engineering and Automation \\ Universidad de Málaga, Spain \\ Email: carlossanchez@uma.es
}

\begin{abstract}
This paper describes a novel electronic nose (enose) aimed at applications that require knowing not only the gas composition and concentration, but also its temporal and spatial evolution. This is done by capturing additional information related to the chemical substance such as the timestamp and geo-location of the measurements, as well as other physical magnitudes of the environment like temperature and humidity for correcting and interpreting the data. The device has been conceived following a modular architecture as a set of independent smart modules, which are interconnected and controlled through an $I^{2} C$ interface by a central processing unit. Each smart module can identify itself, store settings for autoconfiguration and perform signal pre-processing of the measured variables. Smart module types include: chemical sensors, communication interfaces, batteries, data storage, GPS, temperature and humidity.
\end{abstract}

\section{INTRODUCTION}

The detection and measurement of chemical substances is essential for a number of applications such as security, leak detection, or pollution monitoring. Among them, an important subset demands the reliable characterization of chemical substances over long periods of time and/or over large areas, constraining the use of classical instruments such as gas chromatographers or mass spectrometers in favor of more portable devices, being e-noses a practical and effective alternative [1], [2].

An e-nose detects and recognizes chemical volatile substances by means of an array of gas sensors and some kind of pattern recognition algorithm [3]. The working principle consists of exposing the air sample to the sensor array, which induces a reversible physical and/or chemical change in the sensing material which causes in turn an associated variation in electrical properties, such as conductivity [4].

Many are the e-nose prototypes developed for the discrimination of complex vapor mixtures in diverse application fields [5]. However, these prototypes are not suitable for applications requiring some additional information apart from the common gas concentration and identification. Applications needing the time-stamp and location of the gas measurements to infer the gas distribution, or the temperature, pressure and humidity for the correction of the samples, demand using the e-nose together with other instruments and devices, thus resulting in inconvenient, non-handheld systems.

Furthermore, most existing e-noses are designed to work for a reduced set of target substances [6], [7]. That is, they are not

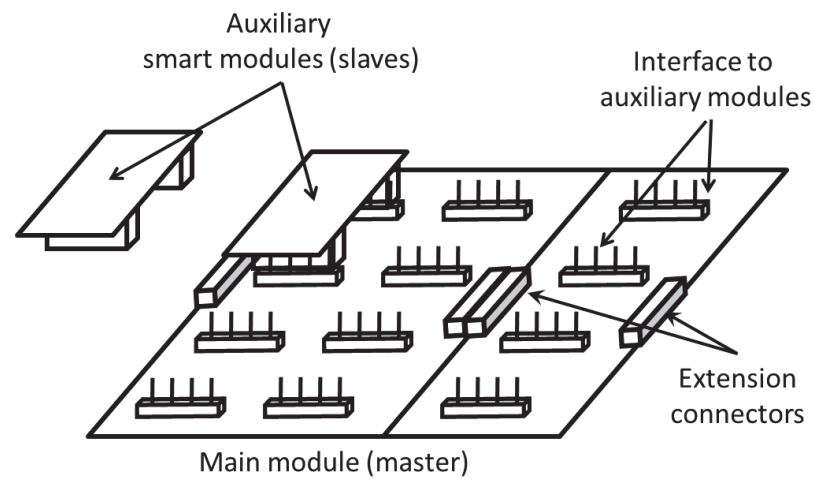

Figure 1. Schematic diagram of the presented e-nose.

adaptable to different applications, needing different e-noses for targeting different compounds.

In this work we describe a novel e-nose conceived upon a configurable architecture which integrates independent, plug\&play, smart modules. This architecture allows for quick and easy adaptation to the application requirements by selecting the appropriate modules (chemical or physical sensors, communication interfaces, batteries, GPS, etc.), while keeping a compact design with reduced dimensions and weight. Furthermore, its ability to geo-reference the gas measurements make it suitable for applications that require spatio-temporal data analysis.

\section{System DESCRIPTION}

The proposed e-nose has been designed as a modular architecture based on the master/slave communication model (see Figure 1). The modular design decomposes the system into smaller parts (i.e. modules) which can then be combined to achieve the desired functionality. Modules are characterized by being scalable, reusable and self-contained functional elements which can be replaced without affecting the rest of the system. Furthermore, the master/slave communication model imposes an unidirectional control of one master, called the Main module, over the slave ones, called Auxiliary modules. Both are equipped with a micro-controller which endows them with certain "intelligence". A description of them is provided next: 


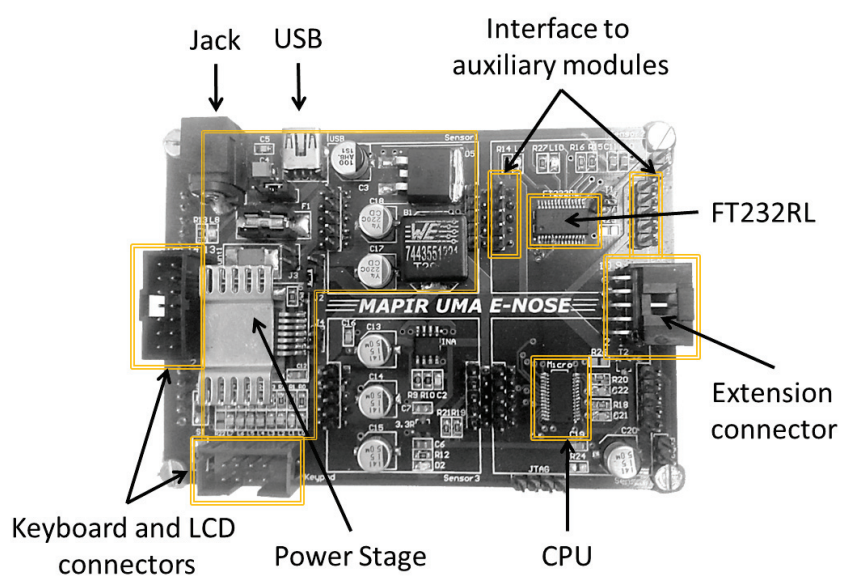

Figure 2. Main module of the e-nose and its components.

\section{A. Main Module}

It is in charge of controlling the auxiliary modules as well as providing the necessary electrical and communication interfaces. Figure 2 depicts its components, which are explained bellow:

- Central processing unit (CPU): It controls the communications with the rest of modules in the architecture and is the responsible of managing all the data collected by the e-nose. First, a temporal reference (time-stamp) is appended to all observations (gas, temperature, location, etc.) to maintain a time-based ordered list, then pairs (time-stamp, observation) are recorded to a rawlog compatible format file [8].

- Power stage: Given the moderate power consumption of some gas sensor technologies, as well as the possibility of hosting a large number of sensors, a dual power input has been considered. For up to $25 \mathrm{~W}$ the e-nose can be powered up using a standard USB mini B connector, from there a jack type connector is needed to provide the necessary electrical power to the system. The regulated voltage of the different components and modules is ensured by means of a switched MCP1702T-3302E/CB voltage regulator with an input range between 8 and 40 volts.

- Interface with auxiliary modules: Apart from the mechanical and electrical connectors necessary to attach and power the slave modules, a communication interface based on the commonly used $I^{2} C$ serial bus is implemented to allow for the interaction between all modules. The $I^{2} C$ bus has been chosen because of its capability to control a network of smart modules with just two general purpose $\mathrm{I} / \mathrm{O}$ pins, something that favors a compact and portable design. Furthermore, the main module is equipped with a 16-pin extension connector for the concatenation of additional $I^{2} C$-based PCB boards supporting further auxiliary modules.

- Interface with external systems: The communication with

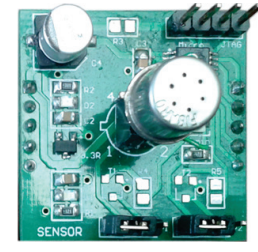

(a)

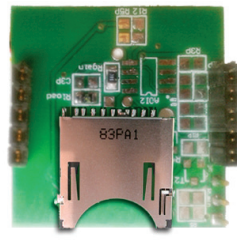

(b)

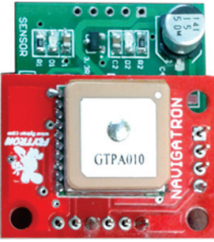

(c)
Figure 3. Example of three smart modules: (a) gas detector module with Figaro gas sensor TGS-2602, (b) SD memory card socket, and (c) GPS module NavigatronV2.

external devices such as computers or tablets is handled by a FT232RL FTDI chip through the same USB mini B type connector used by the power stage. Two are the main functions of this interface: first, it is used to program and configure the settings of the e-nose, and second, it serves to read in real time the data collected by the e-nose.

- Additional connectors: Standard connectors for keyboard, LCD screen and analog/digital inputs/outputs, as well as two power connectors for fans controlled by PWM have been included.

\section{B. Auxiliary Modules}

Auxiliary modules take the role of slaves in the modular architecture of the e-nose. As mentioned above, they are considered smart modules because they are equipped with basic computation capabilities which enable them to be switched on/off at any time (Plug and Play) or to carry out some basic data pre-processing (e.g. signal conditioning, environmental compensation, etc.). This "basic intelligence" of the auxiliary modules is also fundamental for the communication with the main module through $I^{2} C$, endowing the e-nose with autoconfiguration capabilities. The latter refers to the ability of the e-nose to auto-detect the connected auxiliary modules and adapt its operation, a fundamental feature for e-noses that are to be used in wide range of applications.

The main purpose of auxiliary modules is to provide the e-nose with sensing capabilities, although they can also be used to extend its connectivity and functionalities. More precisely, auxiliary modules can be grouped into the following categories:

- Sensors: These modules allocate the sensors, deal with the readings and perform the necessary signal conditioning tasks (filtering, linearization, environmental compensation, etc.) [9], [10]. Sensors hosted within this type of module include: gas sensors (ranging from metal oxide (MOX) or electrochemical (EC), to infrared (IR) technologies), as well as sensors for measuring physical characteristics such as temperature, humidity, pressure or light radiation.

- Communication interfaces: Their task is the communication of the e-nose with any external system required by the application, being wireless interfaces the most interesting ones for portable devices (WiFi, Bluetooh, etc.). 


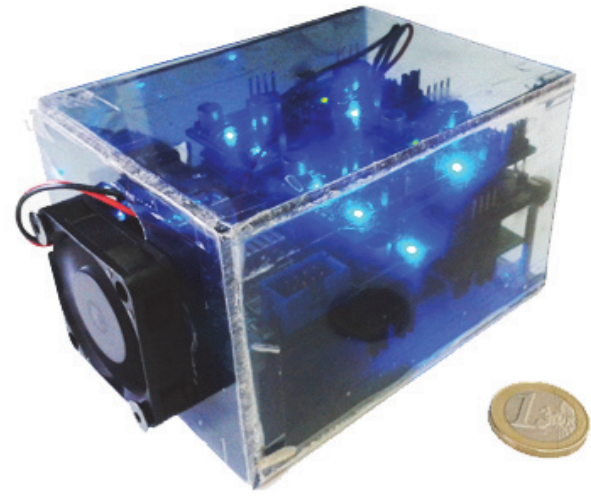

(a)

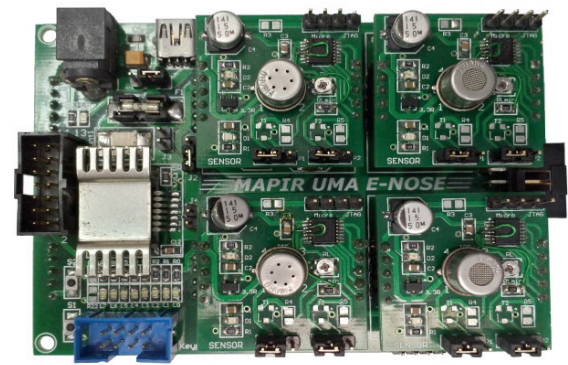

(b)

Figure 4. (a) Picture of the e-nose prototype containing eight smart gas detector modules, a temperature probe, a geo-location module (GPS) and a SD card based memory module. (b) Top view depicting the main module and different auxiliary modules.

- Actuators: These incorporate any actuator to the system, for example, fans, pumps, etc.

- Generic devices: They cover devices such as batteries or memory storage, improving the autonomy and portability of the e-nose.

\section{An Illustrative ApplicAtion}

In this section we depict a specific realization of the presented e-nose and its use in an application where the spatiotemporal data becomes crucial. The objective was to detect, locate and provide the necessary information for the proper monitoring of odorous sources present in a neighborhood of the city of Málaga (Spain). To that end, a prototype of the described e-nose was built and configured to match the application requirements (see Figure 4). The auxiliary modules comprising the prototype are: 8 gas sensor modules (2xTGS-2620, 2xTGS-2611, 2xTGS-2602 and 2xTGS-2600), a temperature, humidity and pressure module for measuring the environmental characteristics that may affect the gas observations, and a GPS (Navigatron V2) to allow the georeference of the detected chemicals. Furthermore, to enhance the portability of the system, it was equipped with a battery (Anker Astro3 12000mAh) and a SD card based memory module. The former is connected to the USB mini B port in the main module (linked to the power stage) to power the e-nose for more than 2 hours. The SD card module is used to store the data in a log file for latter visualization and processing of the measurements. As mentioned in Section II-A, the recorded $\log$ file is compatible with the rawlog format [8], which enables its easy manipulation with open source programs such as RawLogViewer or rawlog-edit, as well as being the input format for some robotic olfaction applications, including gas distribution mapping [11], [12]. The resulting prototype is a compact and lightweight (less than $300 \mathrm{~g}$ ) e-nose, which can be conveniently carried by a person, or a vehicle (e.g. a bike). Furthermore, its modularity allows an easy replacement of any gas sensor in case of malfunction, or if other volatile substances are to be detected.

The most convenient way of visualizing the spatial information from the e-nose is to use a map. Figure 5 shows the map obtained by combining the geo-referenced points provided by the GPS module with the concentrations measured by the gas sensors (color scaled value of the mean among all gas sensors). As can be appreciated, different areas with high volatile concentration (red colors) are detected and localized. However, to better evaluate the temporal response of the gas sensors to the different volatile sources, Figure 6 shows an example of the data collected for three different volatile sources: livestock waste, household garbage and emissions from motor vehicles. Different patterns in the response of the 8 gas sensors suggest the possibility of employing machine learning techniques to perform classification. Pictures of the gas sources are provided by means of an external CMOS camera (not part of the prototype), to enable a better understanding of the displayed data.

\section{CONCLUSION}

In this article, an electronic nose designed for applications that require to perform spatio-temporal analysis of the olfactory data has been presented. It has been conceived as a modular architecture based on smart modules, enhancing the adaptability to the application requirements by selecting the appropriate components. A prototype for the detection of volatile substances in urban areas has been setup, and experimental evaluation demonstrated the suitability of the proposed design.

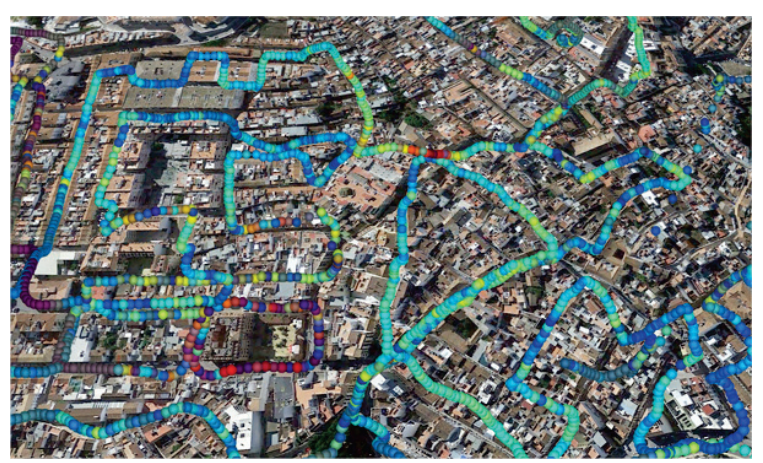

Figure 5. Spatial representation of the e-nose data collected during the inspection of an urban area. A jet color scale is used to represent the measured gas concentration. 


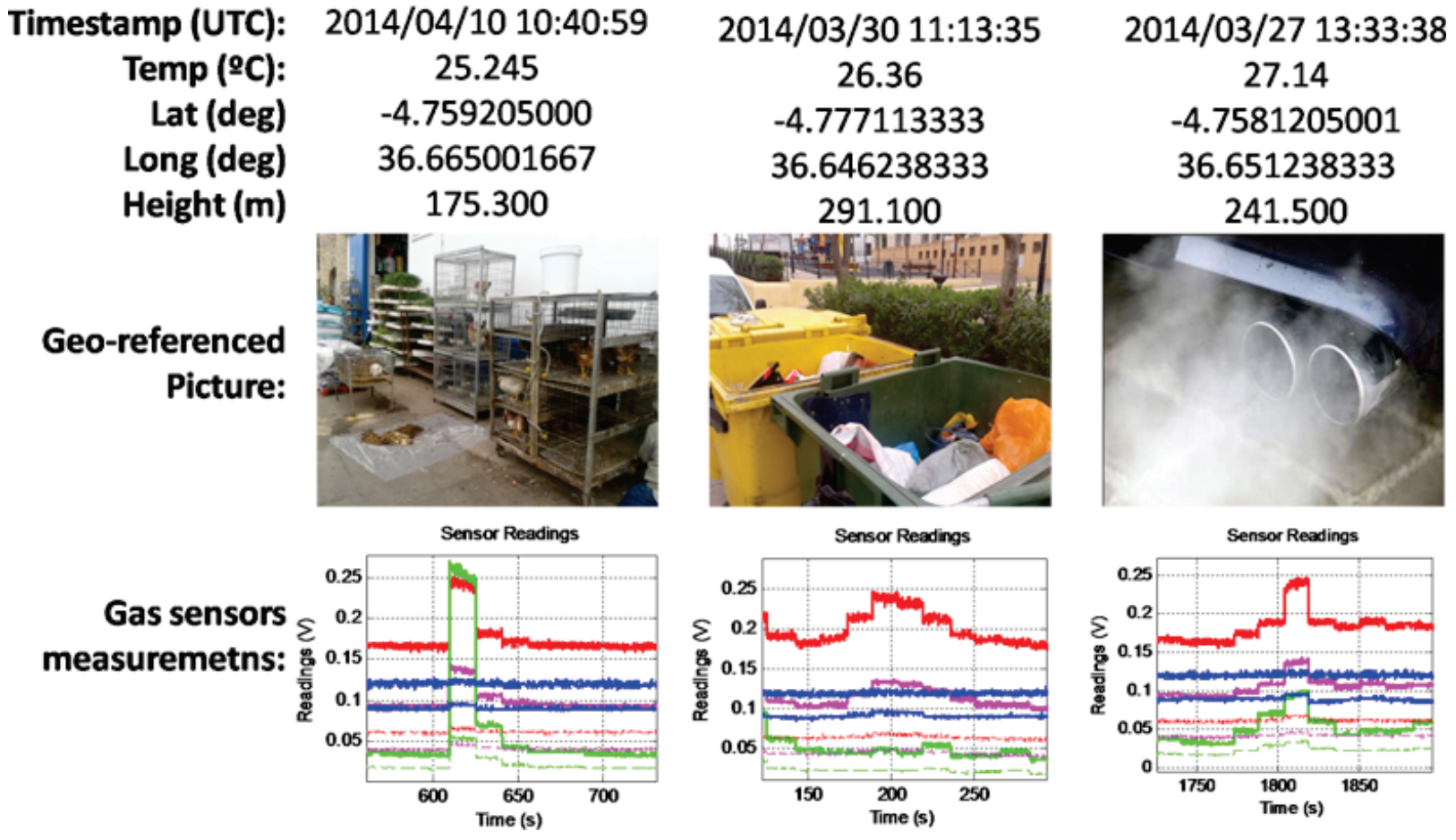

Figure 6. Example of collected data using the proposed e-nose prototype. Top row displays the physical magnitudes related to the measurement point, middle row the images captured by an external CMOS camera (not part of the e-nose prototype), and bottom row the gas sensor measurements.

\section{ACKNOWLEDGMENTS}

This work is supported by the Andalusia Regional Government and the European Union (FEDER) under research projects TEP08-4016 and TEP2012-530.

\section{REFERENCES}

[1] J. G. Monroy, J. Gonzalez-Jimenez, and C. Sanchez-Garrido, "Monitoring household garbage odors in urban areas through distribution maps," in IEEE Sensors, Nov. 2014

[2] A. Romain and J. Nicolas, "Long term stability of metal oxide-based gas sensors for e-nose environmental applications: An overview," Sensors and Actuators B: Chemical, vol. 146, no. 2, pp. 502 - 506, 2010.

[3] A. Bermak, S. Belhouari, M. Shi, and D. Martinez, "Pattern Recognition Techniques for Odor Discrimination in Gas Sensor Array," in The Encyclopedia of Sensors. American Scientific Publishers, 2005.

[4] K. J. Albert, N. S. Lewis, C. L. Schauer, G. A. Sotzing, S. E. Stitzel, T. P. Vaid, and D. R. Walt, "Cross-reactive chemical sensor arrays," Chemical Reviews, vol. 100, no. 7, pp. 2595-2626, 2000.

[5] A. D. Wilson and M. Baietto, "Applications and advances in electronicnose technologies," Sensors, vol. 9, no. 7, pp. 5099-5148, 2009.

[6] J. Lozano, J. Santos, and M. Horrillo, "Classification of white wine aromas with an electronic nose," Talanta, vol. 67, no. 3, pp. $610-616$, 2005.
[7] D. L. Garcia-Gonzalez, N. Barie, M. Rapp, and R. Aparicio, "Analysis of virgin olive oil volatiles by a novel electronic nose based on a miniaturized saw sensor array coupled with spme enhanced headspace enrichment," Journal of Agricultural and Food Chemistry, vol. 52, no. 25 , pp. 7475-7479, 2004.

[8] J.-L. Blanco-Claraco, "Mobile Robot Programming Toolkit (MRPT)," http://www.mrpt.org, 2014.

[9] M. Holmberg and T. Artursson, "Drift compensation, standards, and calibration methods," in Handbook of Machine Olfaction., T. Pearce, S. Schiffman, H. Nagle, and J. Gardner, Eds. Wiley-VCH, 2000, ch. 13 , pp. 326-345

[10] K. R. Kashwan and M. Bhuyan, "Robust electronic-nose system with temperature and humidity drift compensation for tea and spice flavour discrimination," in International Conference on new Techniques in Pharmaceutical and Biomedical Research, Sept 2005, pp. 154-158.

[11] A. J. Lilienthal, M. Reggente, M. Trincavelli, J.-L. Blanco, and J. Gonzalez-Jimenez, "A statistical approach to gas distribution modelling with mobile robots - the kernel $\mathrm{dm}+\mathrm{v}$ algorithm," in IEEE/RSJ International Conference on Intelligent Robots and Systems, IROS., oct. 2009, pp. $570-576$

[12] J.-L. Blanco, J. G. Monroy, J. Gonzalez-Jimenez, and A. J. Lilienthal, "A kalman filter based approach to probabilistic gas distribution mapping," in 28th Symposium On Applied Computing (SAC), mar. 2013, pp. 217 222. 